\title{
Semi-Inclusive $\Lambda$ and $\mathrm{K}_{S}$ Production in $\mathrm{p}-\mathrm{Au}$ Collisions at $17.5 \mathrm{GeV} / \mathrm{c}$
}

I. Chemakin ${ }^{2}$, V. Cianciolo ${ }^{7,8}$, B.A. Cole $^{2}$, R. Fernow ${ }^{1}$, A.D. Frawley ${ }^{3}$, M. Gilkes ${ }^{9}$, S. Gushue ${ }^{1}$, E.P. Hartouni ${ }^{7}$, H. Hiejima ${ }^{2}$, M. Justice ${ }^{5}$, J.H. Kang ${ }^{11}$, H. Kirk ${ }^{1}$, N. Maeda ${ }^{3}$, R.L. McGrath ${ }^{9}$, S. Mioduszewski ${ }^{10}$, D. Morrison ${ }^{10,1}$, M. Moulson ${ }^{2}$, M.N. Namboodiri ${ }^{7}$, G. Rai ${ }^{6}$, K. Read ${ }^{10}$, L. Remsberg ${ }^{1}$, M. Rosati ${ }^{1,4}$, Y. $\operatorname{Shin}^{11}$, R.A. Soltz ${ }^{7}$, S. Sorensen ${ }^{10}$, J.H. Thomas ${ }^{7,6}$, Y. Torun ${ }^{9,1}$, D. Winter ${ }^{2}$, X. Yang ${ }^{2}$, W.A. Zajc ${ }^{2}$, and Y. Zhang ${ }^{2}$,

1 Brookhaven National Laboratory, Upton, New York 11973

2 Columbia University, New York, NY 10027, ${ }^{3}$ Florida State University, Tallahassee, FL 32306,

${ }^{4}$ Iowa State University, Ames, IA 50010, ${ }^{5}$ Kent State University, Kent, OH 44242

${ }^{6}$ Nuclear Science Division, Lawrence Berkeley National Laboratory, Berkeley, CA 94720

7 Lawrence Livermore National Laboratory, Livermore, CA 94550

8 Oak Ridge National Laboratory, Oak Ridge, TN 37831

9 State University of New York at Stony Brook, Stony Brook, NY 11794

10 University of Tennessee, Knoxville, TN 37996, ${ }^{11}$ Yonsei University, Seoul 120-749, Korea

(November 7, 2018)

The first detailed measurements of the centrality dependence of strangeness production in $\mathrm{p}$ - $\mathrm{A}$ collisions are presented. $\Lambda$ and $\mathrm{K}_{S} d n / d y$ distributions from $17.5 \mathrm{GeV} / \mathrm{c}$ - $\mathrm{Au}$ collisions are shown as a function of "grey" track multiplicity and the estimated number of collisions, $\nu$, made by the proton. The $\nu$ dependence of the $\Lambda$ yield deviates from a scaling of p-p data by the number of participants, increasing faster than this scaling for $\nu \leq 5$ and saturating for larger $\nu$. A slower growth in $\mathrm{K}_{S}$ multiplicity with $\nu$ is observed, consistent with a weaker $\nu$ dependence of $\mathrm{K} \overline{\mathrm{K}}$ production than $Y K$ production. 
Significant effort has been devoted in the last decade to measuring strange particle production in nucleusnucleus (A-A) collisions, motivated by predictions that quark-gluon plasma (QGP) formation could enhance strangeness 11,2]. Experiments at both the Brookhaven National Laboratory (BNL) AGS and the CERN SPS accelerators have reported large increases in relative strange particle yields in central light $(\mathrm{Si}, \mathrm{S})[3]$ [6] heavy ion $(\mathrm{Au}, \mathrm{Pb})$ [7 11 induced collisions compared to p-p collisions. However, we still cannot claim a true understanding of the physics of strangeness enhancement due to the complexity of the hadronic interactions underlying A-A collisions and the competing mechanisms proposed and/or used in models 12 th the explain the data.

The difficulty in interpreting the A-A data and the observation that the enhancement is already present in light-ion collisions suggest the use of $\mathrm{p}-\mathrm{A}$ collisions to study this problem further. The simpler final state of p-A collisions may allow the production rate for strange particles to be directly connected with the scattering dynamics of the incoming proton. Previously published $\mathrm{p}-\mathrm{A}$ data 15,16] demonstrated an increase in the inclusive $\mathrm{K} / \pi$ ratio with increasing $\mathrm{A}$ at the AGS, suggesting that the strangeness enhancement mechanism is already at work in p-A collisions. A more thorough analysis [17] of inclusive data at higher energies [18] suggested no overall strangeness enhancement but a possible modest enhancement in $\Lambda$ production offset by a decrease in $\overline{\mathrm{K}}$ production. This result has led to claims that the observed strangeness enhancement in A-A collisions at SPS energies may result from QGP formation [19]. However, extrapolations to central A-A data from inclusive p-A data are intrinsically flawed: Centrally selected A-A events necessarily involve more scatterings of the participant nucleons, and the dynamics of strangeness production may be quite sensitive to these additional scatters. A resolution of this problem requires a detailed measurement of the centrality dependence of strangeness production in p-A collisions.

In this paper we present the first such measurement, made by BNL experiment 910 at the AGS accelerator. The data consist of $\Lambda$ and $\mathrm{K}_{S}$ rapidity spectra and integrated yields obtained as a function of "grey" track multiplicity from p-Au collisions at a beam momentum of $17.5 \mathrm{GeV} / \mathrm{c}$. E910's nearly complete rapidity coverage allows us to accurately estimate the total $\Lambda$ and $\mathrm{K}_{S}$ multiplicities and study the variation of absolute yields with $\nu\left(N_{\text {grey }}\right)$, the estimated number of collisions of the proton in the Au nucleus. A common benchmark «, 20, 11 for evaluating strangeness enhancement in A-A collisions is the scaling of $\mathrm{p}-\mathrm{p}$ data by the number of participants, $N_{\text {part }}$, in the collision. For p-A collisions, an $N_{\text {part }}$ scaling of p-p data would yield [21]

$$
N_{\text {prod }}=\frac{1}{2} N_{\text {prod }}^{\mathrm{pp}}(1+\nu)
$$

since there are two participants in p-p collisions. We com- pare our multiplicities to the yields expected from Eq. 1 to evaluate whether we see enhanced strange particle production using the same benchmark as in A-A collisions. We observe that for $\Lambda$ production Eq. 1 is physically sensible since baryon number is conserved and $\mathrm{B} \overline{\mathrm{B}}$ processes are negligible at our energies. Deviations from Eq.1 would imply contributions from target nucleons not directly struck by the projectile or changes in the probability of participants to fragment into $\Lambda$ 's. E910 was staged in the Multi-Particle Spectrometer (MPS) facility at the AGS. For this data, the Cherenkov tagged secondary proton beam had a mean momentum of $17.5 \pm 0.1 \mathrm{GeV} / \mathrm{c}$ and a $1.5 \%$ momentum spread. The E910 spectrometer was previously described in 22,23]; the results presented here rely on the EOS time projection chamber and the beamline/trigger counters. A $3.9 \mathrm{~g} / \mathrm{cm}^{2} \mathrm{Au}$ target was located $20.5 \mathrm{~cm}$ upstream of the active area of the TPC and was immediately followed by a $10 \mathrm{~cm} \times 10 \mathrm{~cm}$ two-layer scintillating-fiber hodoscope. This multiplicity detector provided two triggers, a minimum-bias (MB) trigger that required 2 hits on each layer and a "central" trigger that required a total of 20 hits in the two layers and selected approximately the $25 \%$ most central events. Due to the low light yield of the fibers the MB trigger suffered significant efficiency loss for low multiplicity events with no highly ionizing tracks.

After finding and fitting the recorded pulses in the TPC we obtained typical resolutions of $0.7 \mathrm{~mm}$ (vertical) and $0.5 \mathrm{~mm}$ (horizontal) for position measurements in each sample. The momentum resolution for particles bending in the $0.5 \mathrm{~T}$ magnetic field varied from $1.2 \%(p<$ $2 \mathrm{GeV} / \mathrm{c})$ to $5.5 \%(p \sim 17 \mathrm{GeV} / \mathrm{c})$. The $d E / d x$ measurement, obtained from a truncated-mean of the TPC samples on each track, provided a resolution of $\sigma /\langle d E / d x\rangle$ $=6 \%$ for typical track lengths. $\Lambda$ 's and $K_{S}$ 's were measured and identified through a combination of topological reconstruction and $d E / d x$ identification of the decay daughters. We paired and removed conversion electrons and positrons, the dominant source of background, with an efficiency of $\approx 50 \%$. We further reduced background from conversions and from false vertices by applying tighter geometric cuts to small opening-angle pairs. We attempted topological fits on $+/$ - track pairs satisfying applied geometric and $d E / d x$ cuts and accepted as $\mathrm{V}_{0}$ candidates those passing applied $\chi^{2}$ cuts with origin $\geq 3.5 \mathrm{~cm}$ from the target. We used a combined likelihood from the single track $d E / d x$ 's and hypothetical $p \pi^{-}$and $\pi^{+} \pi^{-}$invariant masses $\left(M_{\text {inv }}\right)$ of the pairs to identify the decaying particle, obtaining the $M_{\text {inv }}$ distributions shown in Fig. 11. We obtained mass resolutions of $F W H M=4 \mathrm{MeV}, 13 \mathrm{MeV}$ for $\Lambda$ 's and $\mathrm{K}_{S}$ 's, respectively and corresponding $\mathrm{S} / \mathrm{B}$ ratios of $35: 1$ and 30:1. The acceptances were calculated via GEANT simulations of the detector response to $20 \mathrm{M}$ pure $\Lambda$ and $10 \mathrm{M}$ pure $\mathrm{K}_{S}$ decays [24]. We show in Fig. 1] the $y-p_{\perp}$ regions with $>10 \%$ acceptance. For the centrality measurement, we identified as "grey" tracks protons and deuterons in the momentum ranges $[0.25,1.2] \mathrm{GeV} / \mathrm{c}$ and $[0.5,2.4] \mathrm{GeV} / \mathrm{c}$, 
respectively. We obtain the multiplicity of grey tracks, $N_{\text {grey }}$, within our geometric acceptance event-by-event and from this quantity estimate the mean number of collisions suffered by the beam proton, $\bar{\nu}\left(N_{\text {grey }}\right)$, using an established technique [23].

The data presented in this paper resulted from a combined $4.65 \mathrm{M}$ triggers. We required a valid event to have at least one secondary charged particle in the final state and a $\Sigma p_{\perp}>85 \mathrm{MeV} / \mathrm{c}$. In addition, we vetoed one and two-track events containing a high-momentum positive track consistent with a quasi-elastically scattered proton. After applying quality and the above interaction cuts we obtained $2.97 \mathrm{M}$ events, $1.88 \mathrm{M}$ minimumbias and 2.07M central. From these, we reconstructed a total of $156.8 \mathrm{k} \Lambda$ 's and $76.8 \mathrm{k} \mathrm{K}$ 's. Using beam-triggered events we determined trigger efficiency corrections as a two-dimensional function of charged-particle multiplicity and $N_{\text {grey }}$. The correction for multiplicity 1,2 events with $N_{\text {grey }}=0$ is large (5.1) while the average correction for all interactions is 1.1 .

We calculated $\Lambda$ and $\mathrm{K}_{S}$ yields $\Delta N\left(m_{\perp}, y, M_{\text {inv }}\right)$ per event as a function of $N_{\text {grey }}$ after subtracting the $\Lambda$ and $\mathrm{K}_{S}$ background in each bin. We corrected these for acceptance $(\mathrm{A})$, trigger efficiency $(\varepsilon)$, and branching ratio (BR) to obtain an invariant differential yield,

$$
\frac{d^{2} n_{\Lambda / K_{s}}}{m_{\perp} d m_{\perp} d y}=\frac{1}{m_{\perp}} \frac{1}{A \varepsilon \mathrm{BR}} \frac{\Delta N_{\Lambda / K_{s}}\left(m_{\perp}, y\right)}{N_{\mathrm{evt}} \Delta m_{\perp} \Delta y} .
$$

For $N_{\text {grey }}<4$, we used only MB triggers, but for larger $N_{\text {grey }}$ we combined both triggers, weighting by the number of events in each sample. The $m_{\perp}$ spectra are uniformly well-described by exponential distributions except at the highest $m_{\perp}$ values $\left(m_{\perp}-m>0.6\right.$ at mid-rapidity) where we do not have sufficient statistics to accurately determine the background subtraction. We fit the $m_{\perp}$ spectra excluding these points to the form,

$$
\frac{1}{2 \pi m_{\perp}} \frac{d^{2} n}{d m_{\perp} d y}=\frac{1}{2 \pi\left(m_{0}+T\right) T} \frac{d n}{d y} e^{-\left(m_{\perp}-m_{0}\right) / T} .
$$

where $d n / d y$ is a direct parameter of the fit representing the integral of Eq. 3 over $m_{\perp}$. The fits give inverse slopes, $T$, that vary from $0.05 \mathrm{GeV} / \mathrm{c}$ at low and high $y$ to $0.14 \mathrm{GeV} / \mathrm{c}$ at mid-rapidity, consistent with proton spectra obtained from p-A collisions at a similar energy [16. For $N_{\text {grey }}$ bins where we do not have enough statistics to perform the fits we directly sum over $m_{\perp}$ to obtain $d n / d y$. Fig. 2 shows the resulting $d n / d y$ distributions for a sub-set of the available $N_{\text {grey }}$ bins. We estimate 90\% CL point-to-point systematic errors in the $\Lambda$ and $\mathrm{K}_{S} d n / d y$ measurements, including contributions from the fitting, to be $<5 \%$ except for the lowest rapidity bin $(20 \%)$, and estimate the normalization systematic error to be $\pm 10 \%$.

We observe that with increasing $N_{\text {grey }}$, the $\Lambda$ and $\mathrm{K}_{S}$ yields decrease at high rapidity and increase at low rapidity. For $\Lambda$ 's, the decrease in yield at large rapidity is a direct consequence of the increased "stopping" of the projectile baryon resulting from the multiple interactions in the target nucleus 22]. The strong increase in production of strange particles at low rapidity is qualitatively consistent with previously observed trends in secondary particle production in p-A collisions [16,25,26. We show in Fig. 3 the integrated yields that we have obtained by summing our measured $d n / d y$ values as a function of $\nu$. The error on the in-acceptance yield is dominated by and taken to be the same as our uncertainty in the absolute normalization $\pm 10 \%$ at $90 \% \mathrm{CL}$. We show (solid line) in Fig. 3 the expectations from Eq. 1 with $N_{p p}^{\Lambda}=0.054 \pm 0.002$ (syst) and $N_{p p}^{K_{s}}=0.035 \pm 0.002$ (syst) obtained by parameterizing the $\sqrt{s}$ dependence of $\Lambda$ and $\mathrm{K}_{S}$ multiplicities [27] and interpolating to our energy. The $\Lambda$ yields initially increase faster with $\nu$ than expected from the $N_{\text {part }}$ scaling of p-p yields and then saturate and start to decrease. The $\mathrm{K}_{S}$ yields behave similarly with a slower initial increase. The apparent decrease of the yields may result from the fact that we miss a larger fraction of the total $\Lambda$ and $\mathrm{K}_{S}$ yield with increasing $N_{\text {grey }}$ or $\nu$ due to our low-rapidity cut-off. We have estimated the missing yield by fitting the $d n / d y$ distributions to gamma distributions as shown in Fig. 3 and extrapolating these into the unmeasured region to produce the estimated total yields shown in Fig. 3. The uncertainty in the total yield is largest for the larger $N_{\text {grey }}$ or $\nu$ bins where the $d n / d y$ distribution peaks near the edge of our acceptance. We show in Fig. 3 90\%CL systematic errors on the yields with larger errors on the high side to account for the possibility that an unknown mechanism may produce a larger $\Lambda$ yield below $y=0$ than we estimate. The resulting total $\Lambda$ yields shown in Fig. 3 saturate at large $\nu$ and remain flat. We have fit the extrapolated $\Lambda$ yields to an empirical function,

$$
N_{\Lambda}=N_{p p}^{\Lambda}\left(1-e^{-\kappa \nu^{\alpha}}\right) /\left(1-e^{-\kappa}\right),
$$

where $N_{p p}$ is the $\Lambda$ multiplicity in p-p collisions. The obtained function with $\kappa=0.299 \pm 0.008$ and $\alpha=1.29 \pm$ 0.03 describes both the initial rapid rise of the yield and the saturation at large $\nu$. To evaluate the significance of this fast initial increase in the $\Lambda$ yield we plot in Fig. 33 the yield that would result from a "binary-collision" scaling of p-p data,

$$
N^{\mathrm{BC}}(\nu)=N_{p p} \nu
$$

which we view as the fastest plausible increase that could be expected from the multiple scattering of the incoming proton. The $\Lambda$ yields are consistent with this "upper limit" for $\nu \leq 3$ indicating a rate of increase in $\Lambda$ yield with $\nu$ in this region that is approximately twice that given by Eq. 1. We note that the systematic error on the $\nu$ scale of $\pm 15 \%$ [23] is small compared to this difference in slope.

We observe that the $\mathrm{K}_{S}$ yield increases more slowly with $\nu$ than the $\Lambda$ yield and appears to decrease slightly at large $\nu$ even after we have accounted for the missing 
yield. The difference in behavior between the $\Lambda_{-}$and $\mathrm{K}_{S}$ yields may result from the mixture of $\mathrm{K}^{0}$ and $\overline{\mathrm{K}}^{0}$ in the $\mathrm{K}_{S}$ and the fact that these are produced through different processes. In p-p collisions at comparable energies, $\frac{1}{2}$ of the $\mathrm{K}_{S}$ are produced as $\mathrm{K}^{0}$ 's in association with hyperons with the other half produced as $\overline{\mathrm{K}}^{0}$ 's associated with kaons [28]. If we assume that this proportion is not modified in $\mathrm{p}-\mathrm{A}$ collisions and that the total hyperon yields increase in proportion to the $\Lambda$ yield, we can estimate the $\overline{\mathrm{K}}^{0}$ component of the measured $\mathrm{K}_{S}$ yields shown in Fig. 3. The $\overline{\mathrm{K}}^{0}$ component appears to increase by a factor of two at $\nu=3$, roughly consistent with the $N_{\text {part }}$ scaling of p-p data shown in Fig. 3, before starting to decrease slowly with $\nu$. While our data suggests an increase in $\mathrm{K} \overline{\mathrm{K}}$ production with $\nu$, because of uncertainties in the above assumptions we cannot make a stronger statement. A forthcoming analysis of $\mathrm{K}^{-}$production will provide clearer insight into this problem.

In conclusion, we have reported on the first detailed investigation of the centrality dependence of strange particle production in $\mathrm{p}-\mathrm{A}$ collisions using grey track multiplicity and the estimated number of collisions of the projectile nucleon to characterize centrality. We have measured $d n / d y$ distributions for $\Lambda$ and $\mathrm{K}_{S}$ that both show a strong backward shift with increasing $N_{\text {grey }}$ and $\nu$. The estimated total $\Lambda$ yields increase with $\nu$ at a rate approximately twice that expected from the $N_{\text {part }}$ scaling of p-p data for $\nu \leq 3$ and saturate for $\nu>5$. As noted above, this violation of $N_{\text {part }}$ scaling implies either that additional nucleons not directly struck by the projectile contribute to $\Lambda$ production or that the probability for one or more of the participants to fragment into a $\Lambda$ increases with $\nu$. We observe a slower but significant increase in $\mathrm{K}_{S}$ multiplicity with $\nu$ that apparently results from different behavior of the $\mathrm{K}^{0}$ and $\mathrm{K}^{0}$ components of the $\mathrm{K}_{S}$. The observed increase in $\mathrm{K}_{S}$ yield is large enough to allow for a statistically significant increase in $\mathrm{K} \overline{\mathrm{K}}$ production with $\nu$ for $\nu \leq 3$ using a reasonable extrapolation of p-p data.

We conclude that at AGS energies, p-A data show a clear violation of a simple $N_{\text {part }}$ scaling of p-p data. This result has clear qualitative implications for use of such scaling for interpreting strangeness yields in A-A collisions. To quantitatively evaluate the potential implications of our results, we assume that the target contribution to the p-Au $\Lambda$ yield grows as $\nu N_{p p} / 2$ and attribute the remainder to the fragmentation of the projectile and/or energy deposition of the projectile in the nucleus. Then, our data show that the "projectile" contribution increases proportional to $\nu$ for $\nu \leq 3$ with a slope that is the same as for the target nucleons. In A-A collisions where both the projectile and target nucleons multiply scatter, this picture implies that the hyperon and associated kaon yields per participant would increase rapidly with the average number of scatters of the participants, $\langle\nu\rangle$, for $\langle\nu\rangle \leq 3$ giving a maximum possible increase in yield per participant of a factor $\sim 3$. This is precisely the behavior seen in the $\mathrm{K}^{+}$production in $\mathrm{Si}-\mathrm{Au}$ and $\mathrm{Au}-\mathrm{Au}$ collisions at the AGS [4] and $\Lambda$ production in $\mathrm{Pb}-\mathrm{Pb}$ collisions at the SPS 11]. In p-A collisions, the enhancement is more modest - a $50 \%$ increase over $N_{\text {part }}$ scaling at $\nu=3$ - simply because the target nucleons scatter only once. As we have shown, however, this modest enhancement may have profound consequences for interpretation of the strangeness enhancement in nuclear collisions. We note that the above picture picture is consistent with the additive quark model [29] used by Kadija et al. [30] to explain the observed strangeness enhancement in light-ion collisions at the SPS. In particular, the increase in the projectile-like component up to $\nu=3$ is exactly what is expected from the additive quark model. Since the saturation of the $\Lambda$ yield for $\nu>5$ is very likely due to the stopping of the incident baryon we predict that at higher energies the $\Lambda$ yield will continue to increase for $\nu>5$.

We wish to thank Dr. R. Hackenburg and the MPS staff, J. Scaduto and Dr. G. Bunce. This work has been supported by the U.S. Department of Energy under contracts with BNL (DE-AC02-98CH10886), Columbia (DE-FG02-86ER40281), ISU (DOE-FG0292ER4069), KSU (DE-FG02-89ER40531), LBNL (DEAC03-76F00098), LLNL (W-7405-ENG-48), ORNL (DEAC05-96OR22464) and UT (DE-FG02-96ER40982) and the National Science Foundation under contract with FSU (PHY-9523974).

[1] J. Rafelski and B. Mueller, Phys. Rev. Lett. 48, 1066 (1982).

[2] P. Koch, B. Müller, and J. Rafelski, Phys. Rep. 142, 167 (1986).

[3] T. Abbott et al., Phys. Rev. Lett. 64, 847 (1990).

[4] L. Ahle et al., Phys. Rev. C60, 044904 (1999).

[5] T. Alber et al., Z. Phys. C64, 195 (1994).

[6] F. Antinori et al., Phys. Lett. B447, 178 (1999).

[7] L. Ahle et al., Phys. Rev. C58, 3523 (1998).

[8] S. Ahmad et al., Phys. Lett. B382, 35 (1996).

[9] C. Bormann et al., J. Phys. G G23, 1817 (1997).

[10] S. Margetis et al., J. Phys. G G25, 189 (1999).

[11] E. Andersen et al., Phys. Lett. B449, 401 (1999).

[12] R. Matiello et al., Phys. Rev. Lett. 63, 1459 (1989).

[13] Y. Pang, T. Schlagel, and S. Kahana, Phys. Rev. Lett. 68, 2743 (1992).

[14] P. Braun-Munzinger and J. Stachel, Nucl. Phys. A606, 320 (1996).

[15] T. Abbott et al., Phys. Rev. Lett. 66, (1991).

[16] T. Abbott et al., Phys. Rev. D 45, 3906 (1992).

[17] M. Gazdzicki, Nucl. Phys. A566, 503C (1994).

[18] H. Bialkowska et al., Z. Phys. C55, 491 (1992).

[19] M. Gazdzicki, J. Phys. G G23, 1881 (1997).

[20] E. Andersen et al., Phys. Lett. B433, 209 (1998).

[21] The projectile counts as one participant no matter how many times it scatters. 
[22] I. Chemakin et al., (1999), nucl-ex/9902009, submmitted to PRL.

[23] I. Chemakin et al., Phys. Rev. C 60, 024902 (1999).

[24] We have neglected small $(<5 \%)$ multi-track losses.

[25] J. Elias et al., Phys. Rev. D 22, 13 (1980).

[26] C. DeMarzo et al., Phys. Rev. D 29, 2476 (1984).

[27] M. Gazdzicki and D. Rohrich, Z. Phys. C71, 55 (1996).

[28] K. Alpgård et al., Nucl. Phys. B103, 234 (1976).

[29] A. Bialas, W. Czyz, and W. Furmanski, Acta Phys. Polon. B8, 585 (1977).

[30] K. Kadija et al., Z. Phys. C66, 393 (1995).
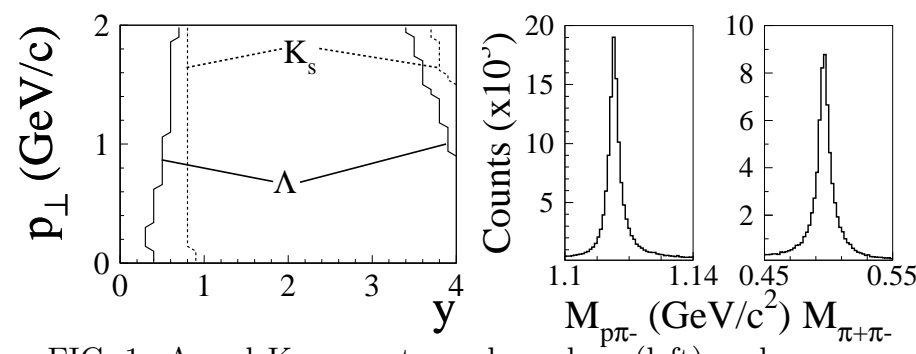

FIG. 1. $\Lambda$ and $\mathrm{K}_{S}$ acceptance boundary (left) and reconstructed invariant mass spectra (right).
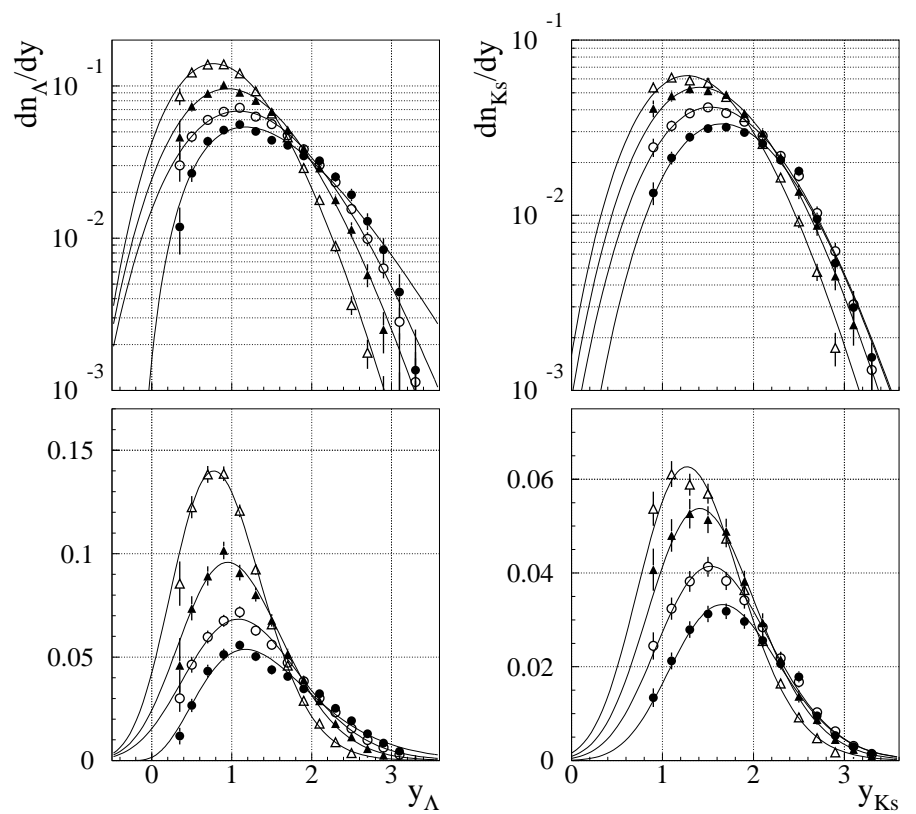

FIG. 2. $\Lambda$ (left) and $\mathrm{K}_{S}$ (right) rapidity density distributions for different $N_{\text {grey }}$ plotted in both log (top) and linear(bottom) scale. $N_{\text {grey }}=\bullet-0, \circ-1, \nabla-2$ and $\nabla-4$.
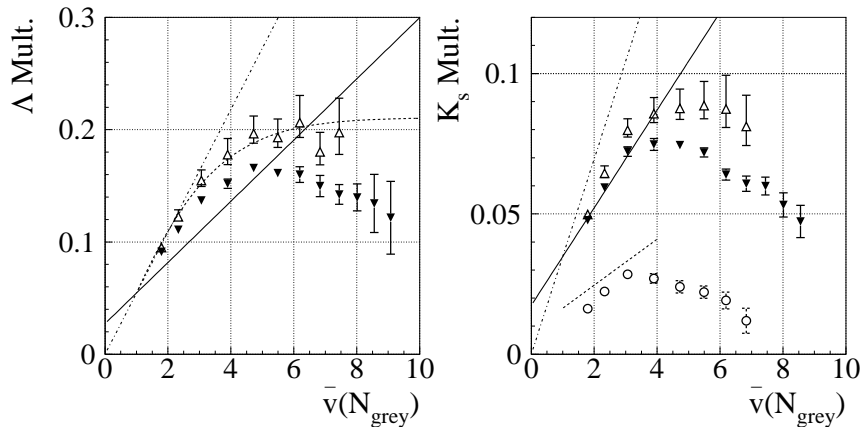

FIG. 3. $\Lambda$ (left) and $\mathrm{K}_{S}$ (right) multiplicities vs $\bar{\nu}\left(N_{\text {grey }}\right)$. $\boldsymbol{\nabla}$ - in E910 acceptance, $\Delta$ - total yield (syst. errors from extrapolation included), ○- est. $\overline{\mathrm{K}}^{0}$ component of $\mathrm{K}_{S}$. Lines: Solid - $N_{\text {part }}$ scaling, dot-dashed - Binary collision, dashed empirical fit to $\Lambda$ (left panel, see text for details), est. $N_{\text {part }}$ scaling for $\overline{\mathrm{K}}^{0}$ (right panel). 